\title{
Condom use by female sex workers and their clients in Mexico: who decides and does it matter?
}

\author{
Annick Bórquez, Timothy B Hallett, Gabriela B Gomez, Geoffrey P Garnett
}

- Additional figures, tables and appendix are published online only. To view these files please visit the journal online (http://sti. bmj.com).

Department of Infectious Disease Epidemiology, Imperial College, London, UK

\section{Correspondence to} Department of Infectious Disease Epidemiology, Faculty of Medicine, Imperial College London, St Mary's Campus, Norfolk Place, London W2 1PG, UK; annick.borquez06@ imperial.ac.uk

Accepted 14 December 2010 Published Online First 26 January 2011

\section{ABSTRACT}

Objectives To explore the effect of different patterns of condom use by clients and female sex workers (FSW) on HIV transmission in Mexico.

Methods Data from 2401 face-to-face interviews among FSW from Morelos and Michoacán in Mexico were used to build and parameterise a deterministic model of HIV transmission between FSW, their stable partners and clients.

Results For the observed patterns of condom use among FSW, a range of patterns of use among clients would be consistent. Two extreme patterns were explored: either clients conformed to FSW decision, or they had a strict preference for bought sex with or without condoms. HIV prevalence in the model is greater in the first of these scenarios.

Conclusions Patterns of condom use between sex workers and clients are an important, but hidden, determinant of epidemic spread. More detailed information on condom use negotiation and behavioural patterns of clients is needed to understand the potential for transmission in these populations and in order to direct intervention efforts more effectively.

In Mexico and other countries with concentrated HIV epidemics, targeting high-risk groups has been a focus of sexually transmitted infection control efforts. ${ }^{1}{ }^{2}$ An important determinant of the extent of HIV spread will be the heterogeneity in risk behaviour within these groups. Mathematical models using data on behaviour allow us to predict the risks of HIV spread and the impact of interventions. While information on individuals' own behaviour can be recorded, information on partners' risks is less readily captured. In fact, in many cases, several different patterns of behaviour can be consistent with the same individual-level behavioural reports. In this paper, we test the hypothesis that patterns of mixing and condom use among sex workers and their clients are a determinant of the potential extent of epidemic spread.

\section{METHODS}

A deterministic mathematical model was developed that represents heterosexual transmission of HIV between FSW, their clients and non-commercial partners (see supplementary figure S-1, available online only). We focused on the heterogeneity in behaviour between FSW categorising them into eight mutually exclusive sexual activity classes defined by: (1) condom use at last sex; (2) number of clients per week; and (3) having a noncommercial partner (see supplementary table S-1, available online only). We parameterised the model using data from a behavioural survey undertaken in Morelos and Michoacán, Mexico, where 2401 FSW participated in a validation study, part of the second generation surveillance implemented by the Instituto Nacional de Salud Pública (INSP) and Centro Nacional para la Prevención y el Control del VIH/SIDA (CENSIDA) in 2001 (see supplementary table S-2, available online only). The model is described in detail in the supplementary material (model equations S-1 available online only).

Based on those data, it was assumed that some FSW always use condoms (84\%) and some never do $(16 \%)$. Then, the effect of the mixing pattern between clients and FSW was explored by simulating an HIV epidemic under two scenarios. In one scenario (figure 1A) 'homogeneous client population', it was assumed that condom use was determined by FSW only (ie, clients chose FSW randomly, such that clients used condoms in some transactions and not others). In the alternative scenario (figure 1B) 'heterogeneous client population', it was assumed that condom use also depended on clients' preferences (ie, some clients always used condoms and some never did, and each type of client sought FSW who matched their behaviour). The contribution of each subpopulation to the HIV epidemic was also evaluated through sensitivity analyses by measuring changes in incidence according to the frequency of condom use with both clients and partners, and the number of clients.

\section{RESULTS}

HIV epidemics were simulated under the two scenarios. In the homogenous client scenario (figure 1C), the incidence in the total population was greater than in the heterogeneous client scenario (figure 1D), peaking at two versus approximately 0.4 cases per 100 person-years at risk. Figures 1E,F compare the incidence at equilibrium among FSW, their clients and non-commercial partners. In the homogenous client scenario, the most affected groups were FSW who did not use condoms and their stable partners as well as all clients (figure 1E). Among FSW who used condoms with clients, HIV incidence was 15 times lower than among FSW who did not. In the heterogeneous client scenario (figure 1F), FSW and clients who used condoms did not get infected. Incidence among FSW and clients who did not use condoms was similar to the rate in the homogenous client scenario. The higher overall incidence under the homogenous client scenario is largely because the risk of infection experienced by clients who do not use condoms in the heterogeneous scenario is shared with all the clients in the homogenous client unlocked scheme, see http://sti. bmi.com/site/about/unlocked. xhtml 
Figure 1 HIV prevalence and incidence among all groups and incidence at equilibrium by population subgroup assuming two mixing patterns between FSW and Clients: (A) Homogeneous client population (Hom-CP) scenario, where all clients (square) mix randomly with FSW (circles) who either always or never use condoms. (B) Heterogeneous client population (Het-CP) scenario, where clients are divided into condom users (dark square) and non-users (light square) and mix assortatively with FSW according to condom use. (C) and (D) show the HIV prevalence in light grey and incidence in black among all groups for the Hom-CP and Het-CP scenario, respectively. $(E)$ and $(F)$ show the incidence at equilibrium by subpopulation for the Hom-CP and Het-CP scenario, respectively. FSW-C: FSW who consistently use condoms; FSW-NC: FSW who never use condoms; Clients: clients of FSW who use condoms only with FSW who use condoms (Hom-CP only); Clients-C: clients who consistently use condoms and only have sex with FSW who use condoms (Het-CP only); Clients-NC: clients who never use condoms (Het-CP only); Partners-C: non-paying partners of FSW who always use condoms: Partners-NC: non-paying partners of FSW who never use condoms. (All model parameters are provided in the supplementary appendix, available online only).

scenario, and second, that the clients in the homogenous scenario act as a bridge to distribute infections to FSW who do use condoms.

In sensitivity analyses decreases in condom use were associated with increased HIV incidence in the homogeneous client scenario but not in the heterogeneous client scenario until a threshold level of unprotected sex allowed HIV spread. The same pattern was observed for increases in contact rates among FSW (see supplementary figure S-2, available online only). These results suggest that continued epidemic spread is more sensitive to changes in behaviour when there is a homogenous client population.

\section{DISCUSSION}

This modelling exercise highlights the importance of mixing patterns with regard to condom use in determining the scale of HIV epidemics and the impact of changes in risk behaviour on transmission. Different prevention strategies may be appropriate to control HIV infection depending on these patterns. For a heterogeneous client population, interventions could be efficiently targeted to those particular FSW and clients who do not use condoms, while in the homogenous mixing scenario interventions would have to include strong campaigns for all clients to adopt condom use in order to reduce the bridging of transmission between the two groups of FSW. The only available data that could allow us to make hypotheses about which mixing scenario would better approximate the sex industry in Mexico come from US border cities. These suggest there is a group of clients that refuses to use condoms and generally buys sex from FSW who are also more likely not to use condoms. ${ }^{3-6}$ However, this is a very particular context making it difficult to generalise the findings.

The classification of FSW and the mixing patterns simulated in this report are extreme ends of a continuous range of behavioural

\section{Key messages}

- Reported levels of condom use among FSW could translate in different HIV epidemics depending on the clients' patterns of use and mixing.

- Consistent condom use by particular clients generates lower HIV prevalence than if the same inconsistent usage of condom is shared by all clients.

- Information on condom use negotiation among clients of FSW would be valuable for modelling, surveillance and programmatic purposes.

patterns. Among other complex aspects of sexual behaviour that are only partly accessible through egocentric survey data, ${ }^{7} 8$ these differences in mixing patterns may be an important reason why behavioural interventions do not always translate to substantial changes in sexually transmitted infection incidence, and why impact may vary between settings. Collecting information on within-person variation in condom use in addition to that more usually collected on between-individual variation, could lead to a better understanding of transmission in these populations, while a further characterisation of clients' negotiation of condom use could strengthen interventions.

Acknowledgements The authors would like to thank the Instituto Nacional de Salud Pública (INSP) and the Centro Nacional para la Prevención y el Control del VIH/ SIDA (CENSIDA), México, for sharing these data as well as all the participants in the behavioural survey for their time and collaboration.

Funding This study was funded by the Medical Research Council and the Wellcome Trust.

Competing interests None

Patient consent Obtained 
Ethics approval This study was conducted with the approval of the scientific, ethical and biosecurity boards of the National Institute of Public Helath in Mexico (INSP).

Contributors $A B$ and GPG designed the study. $A B$, TBH and GBG performed the analyses. All authors contributed to the interpretation of results and write up.

Provenance and peer review Not commissioned; not externally peer reviewed.

\section{REFERENCES}

1. Gayet CMC, Sacknoff D, Guli L. Prácticas sexuales de las poblaciones vulnerables a la epidemia de VIH/SIDA en México. Serie Angulos del SIDA. México: CENSIDA, Facultad Latinoamericana de Ciencias Sociales, Sede México, 2007.

2. Aral SO. Behavioral aspects of sexually transmitted diseases: core groups and bridge populations. Sex Transm Dis 2000;27:327-8.
3. Bucardo J, Semple SJ, Fraga-Vallejo M, et al. A qualitative exploration of female sex work in Tijuana, Mexico. Arch Sex Behav 2004;33:343-51.

4. Goldenberg SM, Cruz MG, Strathdee SA, et al. Correlates of unprotected sex with female sex workers among male clients, Tijuana, Mexico. Sex Transm Dis 2010;37:319-24.

5. Larios SE, Lozada R, Strathdee SA, et al. An exploration of contextual factors that influence HIV risk in female sex workers in Mexico: the Social Ecological Model applied to HIV risk behaviors. AIDS Care 2009;21:1335-42.

6. Patterson TL, Goldenberg S, Gallardo M, et al. Correlates of HIV, sexually transmitted infections, and associated high-risk behaviors among male clients of female sex workers, Tijuana, Mexico. AIDS 2009;23:1765-71.

7. Ghani AC, Aral SO. Patterns of sex worker-client contacts and their implications for the persistence of sexually transmitted infections. J Infect Dis 2005;191(Suppl 1):S34-41.

8. Ghani AC, Swinton J, Garnett GP. The role of sexual partnership networks in the epidemiology of gonorrhea. Sex Transm Dis 1997;24:45-56.

\section{Have confidence in your decision making.}
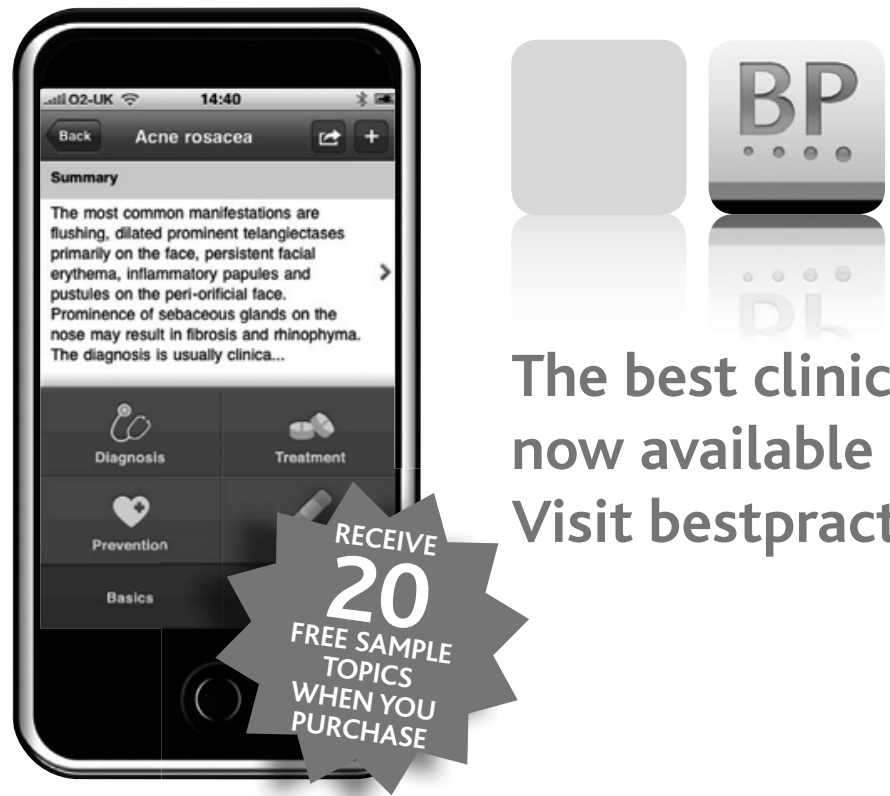

The best clinical decision support tool is now available as an app for your iPhone. Visit bestpractice.bmj.com/app

\section{clinicians $\bullet$ medical students $\bullet$ nurses $\bullet$ healthcare practitioners}

